\title{
Models as a high-risk group: the health implications of a size zero culture
}

\author{
Janet L. Treasure, Elizabeth R. Wack and Marion E. Roberts
}

\section{Summary}

The size zero culture of the fashion industry is damaging not only to the general public but also to the models themselves. This editorial explores mechanisms unique to this population that increase the risk of developing an eating disorder, and discusses the potential for successful intervention by the fashion and beauty industries in the modelling world.

\section{Declaration of interest}

None.
Janet Treasure (pictured) is a consultant psychiatrist for the South London and Maudsley NHS Trust and Professor of Psychiatry at King's College London. She is chief medical advisor for the UK's main eating disorders charity, beat. Elizabeth Wack is a clinical psychology doctoral student at the University of Central Florida in Orlando, Florida, USA and Marion Roberts is a research fellow in the Eating Disorders Research Unit at the Institute of Psychiatry, King's College, London.

There has been widespread concern that the fashion industry, by promulgating ever-diminishing extremes of thinness, is creating a 'toxic' environment in which eating disorders flourish. The Academy of Eating Disorders has written a position statement for the attention of the fashion industry outlining several recommendations to improve both the health of the public and that of models (www.aedweb.org/media/fashion.cfm).

The aim of this editorial is to consider the implications of the fashion industry's expectation of extreme leanness on the models own health and also to set this into the context of public health The direct risks for the models are twofold. First, starvation has a general effect upon all organs in the body, including the brain, and the impact may be profound if the deprivation occurs during development. Second, the demand for, and overvaluation of, extreme thinness within a culture of scrutiny and judgement about weight, shape and eating, increases the risk of developing an eating disorder.

\section{The health consequences of low weight}

There are many health consequences of being underweight. We briefly consider the impact on reproduction, bones and the brain.

Leptin decreases as body weight falls. Without adequate levels of leptin the cascade of hormonal events that controls ovulation and implantation becomes disrupted. Menstruation becomes irregular or absent and fertility is diminished. The Dutch famine in 1944 and the Chinese famine of 1959-1961 were associated with a fall in fertility. In addition, children in utero and beyond had an increased risk of metabolic and reproductive problems and mental illness later in life. ${ }^{1}$ Poor nutrition stunts bone development (in the growth phase) and reduces bone turnover and repair, leading to osteoporosis (the impact on bones in eating disorders is a clear exemplar of these effects). Even minor disturbance in eating behaviour during adolescence is associated with adverse health outcomes later in life. ${ }^{2}$

In humans, the brain accounts for $20 \%$ of an individual's energy expenditure and plays a key role in nutritional homoeostasis. The brain itself shrinks in anorexia nervosa and there is uncertainty as to whether this is fully reversible. The response to starvation includes adjustment of metabolic and physiological processes and changes in drive, thoughts, feelings and behaviour. Starved individuals become preoccupied with food. Keys et al described in great detail subjective and objective reactions to a short period of experimental starvation in men. ${ }^{3}$

\section{Binge priming}

Animal models explain how environmental changes might produce eating disorders. For example, if after a period of food restriction animals are intermittently exposed to highly palatable food, they will significantly overeat. This pattern continues when their weight is restored. ${ }^{4}$ This tendency to overconsume, or 'binge', when exposed to palatable foods remains several months after the period of 'binge priming'. Not only do these animals overeat palatable food but they are also more prone to show addictive behaviours to the more typical substances of misuse, such as alcohol and cocaine. Underpinning these behavioural changes is an imbalance in chemical transmitters in the reward network, for example, dopamine, acetylcholine, endogenous opiates and cannabinoids. The persistent priming of reward circuits by palatable foods resembles the phenomenon of reward sensitisation produced by drug misuse.

Translating into the human situation, we would predict that binge priming caused by irregular dieting and/or extreme food restriction, interspersed with intermittent consumption of snacks and other highly palatable food, might lead to permanent changes in the reward system. Several hypotheses follow from this:

(a) if binge priming occurs in adolescence, when the developing brain is more susceptible to reward, persistent eating problems may follow;

(b) people exposed to binge priming will be more prone to develop substance misuse.

Some empirical evidence supports the first hypothesis in that there are developmental continuities between eating patterns in early life and the later development of eating disorders. For example, people with eating disorders report a higher consumption of high-palatability foods (fast foods and snack foods) and less regular meal times in childhood. Binge eating is persistent, with binge eating disorder present on average for 14 years, and bulimia nervosa for 5.8 years. ${ }^{5}$ Abnormal eating behaviours in early adolescence precede substance misuse ${ }^{6}$ and alcohol use disorders commonly supersede clinical bulimic disorders, ${ }^{7}$ confirming the second hypothesis. 


\section{Models and the risk of eating disorders}

Eating patterns that an individual may have found to be integral in the maintenance of a particular shape during her modelling career may lead to deleterious health consequences and maladaptive eating behaviours that affect her far beyond the typically rather short years of such a career. Furthermore, binge priming might also explain why models have such a high rate of substance misuse. ${ }^{8}$

In addition to the biological factors described above, social factors contribute to the unhealthy lifestyle common among those pursuing a modelling career. Constant exposure to media images depicting thin women reduces body-related self-esteem. A metaanalysis of data from 25 studies found that this effect was most pronounced in adolescents and in participants who valued thinness. ${ }^{9}$ Body-related self-esteem is particularly pertinent in young models as it relates to their career success. Criticism, teasing and bullying focused on food, weight and shape issues increase the risk of developing an eating disorder. Fashion models are frequently judged and evaluated on these domains and critical and hostile comments, under the guise of professional development, will increase the risk of developing eating disorders.

\section{Successful intervention in other domains}

Prevention and regulation of toxic environments is not impossible. Progress has been made in sport and dance. High-performance athletes are also at risk of eating disorders especially in those areas in which excess weight is a handicap or where aesthetic factors are judged. Concerted efforts have been made in the UK to set forth guidelines for high-performance athletes and their coaches in an attempt to reduce the prevalence of eating disorders, unhealthy weight loss and maintenance practices. The UK Sport guidelines ${ }^{10}$ are based on practical strategies that consider the demands of the sport and the long-term health consequences often resulting from those demands.

Following this template, similar approaches to standardisation of care and health for fashion models could be introduced. Unfortunately, such initiatives are yet to be embraced by the fashion industry, as evidenced by the recent inconclusive outcomes from the UK Model Health Inquiry. ${ }^{11}$ As models are embedded within the fashion industry, which holds responsibility for the idealisation of emaciation, it is hoped that the drive for ever more extreme thinness could be stemmed at the source, resulting in benefits for all of society.

\section{The future}

The current fashion for extreme thinness among models unnecessarily puts their physical and psychological health in jeopardy. Starvation disrupts growth and reproductive function and can have profound and persistent effects on brain development. These risks are particularly profound in young women who, in a bingepriming environment, may be more prone to develop other addictive behaviours. Along with an increased risk of substance and alcohol use and misuse, the risk of developing an eating disorder will also be increased. The longer-term health implications on models' bone and reproductive health are unknown but evidence suggests the outcomes are not promising. The recent guidelines from the British Fashion Council, proposing not to include children under 16 years of age as models, is a welcome first step. Might this be taken further (e.g. legislation on age limit for competitive gymnastics)?
Beyond the catwalk, there are wider public health implications. The promotion of the thin ideal, in conjunction with the ready access of highly palatable foods, produces a binge-priming environment. This might explain the exponential increase in eating disorders seen in women born in the last half of the 20th century and in part also contributes to the increase in obesity.

Public health initiatives can be integrated to tackle both of these problems. The fashion and beauty industry can play a key role in preventing the development of unhealthy lifestyles in young people. Indeed, Body Talk, a prevention programme focused on self-esteem developed by Dove in partnership with the UK eating disorder charity beat (http://www.b-eat.co.uk) takes steps to modify the unrealistic 'ideal form' both as displayed in the flesh by fashion models and through the use of digitally enhanced photography. More focus on these issues will decrease unhealthy forms of dieting, dysregulated eating behaviours and body dissatisfaction among young people. Although it may take time to change such an ideal we should not be faint hearted but remember what has similarly been achieved in relationship to cigarette smoking. People are now starting to listen to the abundance of scientific evidence concerning the harm that such images hold not only for those paid to portray it, but for those who pay to emulate it.

Janet L. Treasure, MD, PhD, FRCP, FRCPsych, Elizabeth R. Wack, MSC, Marion E. Roberts, MSC, Institute of Psychiatry, Department of Psychological Medicine and Psychiatry, Section of Eating Disorders, King's College London, UK

Correspondence: Janet Treasure, Department of Academic Psychiatry, 5th Floor Bermondsey Wing, Guy's Hospital, London SE1 9RT, UK. Email: j.treasure@iop.kcl.ac.uk

First received 17 Aug 2007, final revision 28 Oct 2007, accepted 2 Nov 2007

\section{References}

1 Altschuler EL. Schizophrenia and the Chinese famine of 1959-1961. JAMA 2005; 294: 2968.

2 Johnson JG, Cohen P, Kasen S, Brook JS. Eating disorders during adolescence and the risk for physical and mental disorders during early adulthood. Arch Gen Psychiatry 2002; 59: 545-52.

3 Keys A, Brozek J, Henschel A. The Biology of Human Starvation. University of Minnesota Press, 1950.

4 Corwin RL. Bingeing rats: a model of intermittent excessive behavior? Appetite 2006; 46: 11-15.

5 Pope HG Jr, Lalonde JK, Pindyck L, Walsh T, Bulik CM, Crow SJ, McElroy SL, Rosenthal N, Hudson JI. Binge eating disorder: a stable syndrome. Am J Psychiatry 2006; 163: 2181-3.

6 Measelle JR, Stice E, Hogansen JM. Developmental trajectories of cooccurring depressive, eating, antisocial, and substance abuse problems in female adolescents. J Abnorm Psychol 2006; 115: 524-38.

7 Bulik CM, Klump KL, Thornton L, Kaplan AS, Devlin B, Fichter MM, Halmi KA, Strober M, Woodside DB, Crow S, Mitchell JE, Rotondo A, Mauri M, Cassano GB, Keel PK, Berrettini WH, Kaye WH. Alcohol use disorder comorbidity in eating disorders: a multicenter study. J Clin Psychiatry 2004; 65: 1000-6.

8 Santonastaso P, Mondini S, Favaro A. Are fashion models a group at risk for eating disorders and substance abuse? Psychother Psychosom 2002; 71: $168-72$.

9 Groesz LM, Levine MP, Murnen SK. The effect of experimental presentation of thin media images on body satisfaction: a meta-analytic review. Int J Eat Disord 2002; 31: 1-16.

10 UK Sport. Eating Disorders in Sport: A Guideline Framework for Practitioners Working with High Performance Athletes. UK Sport, 2007 (http:// www.uksport.gov.uk/pages/uk_sport_publications/).

11 Model Health Inquiry. Fashioning a Healthy Future: the Report of the Model Health Inquiry, September 2007. Model Health Inquiry, 2007 (http:// www.modelhealthinquiry.com/docs/The\%20Report\%20of\%20the $\% 20$ Model\%20Health\%20Inquiry,\%20September\%202007.pdf). 